\title{
REALIZABILITY INTERPRETATION OF PROOFS IN CONSTRUCTIVE ANALYSIS
}

\author{
HELMUT SCHWICHTENBERG
}

\begin{abstract}
We prove constructively (in the style of Bishop) that every monotone continuous function with a uniform modulus of increase has a continuous inverse. The proof is formalized, and a realizing term extracted. It turns out that even in the logical term language - a version of Gödel's $T$ - evaluation is reasonably efficient.
\end{abstract}

There have been many attempts to formalize constructive analysis as presented in the classic textbook of Bishop (1967). One reason to do this is to uncover the computational content of constructive proofs. Here we describe a rather explicit formalization, from the point of view of later term extraction by a realizability interpretation. In particular, we deal with the existence of a continuous inverse to a monotonically increasing continuous function. The proof uses the Intermediate Value Theorem.

Some optimizations in definitions and proofs are necessary to produce extracted terms that can be evaluated efficiently. These are (a) addition of external code to the definition of arithmetical operations, which is used (based on the corresponding function of the programming language) when the arguments are numerals; (b) introduction of the let-construct in extracted terms; (c) the "non-computational" quantifiers of Ulrich Berger (1993, 2005b).

Related work. The fact that every continuous function with a "modulus of uniform strict monotonicity" has a uniformly continuous inverse has been proved constructively by Mandelkern (1982). A different proof has been given by Kohlenbach (1995); there, the complexity of realizers is discussed, from a rather general point of view. More recently, Josef Berger (2005a) introduced a concept he called "exact representation of continuous functions", and based on this gave a construction converting one such representation of an increasing function into another one of its inverse.

The desire to machine extract the computational content of constructive existence proofs has been one of the motivations of the FTA project Geuvers et al. (2000), where Kneser's constructive proof of the fundamental theorem of algebra was formalized in Coq. This work has recently been extended to build a "Constructive Coq Repository (C-CoRN)" at Nijmegen (Barendregt, Geuvers, Wiedijk, Cruz-Filipe). However, extraction of reasonable programs from proofs in this setup turned out to be problematic. One reason is that witnesses were missing from computational meaningful axioms (e.g., strong extensionality $\left.\forall_{x, y}(f(x) \# f(y) \rightarrow x \# y)\right)$, another one that the Set, Prop distinction in Coq was found to be insufficient (cf. Cruz-Filipe (2004)).

The rest of the paper is organized as follows. In Section 1 we review Kreisel's (modified) realizability interpretation (1959), for an appropriate version of Heyting's arithmetic $\mathrm{HA}^{\omega}$ in finite types. Section 2 presents an 
informal proof of the existence of a continuous inverse function. Section 3 discusses a formalization of this proof, from the point of view of a planned extraction of realizing terms. In Section 4 the extracted terms are presented and discussed, and also some numerical experiments are carried out. Section 5 concludes.

\section{Realizability Interpretation}

We first describe some proof-theoretic background on term extraction, as it is implemented in the Minlog proof assistant (www.minlog-system.de). It is based on modified realizability introduced by Kreisel (1959): from every constructive proof $M$ (in natural deduction) of a formula $A$ with computational content one extracts a term $\llbracket M \rrbracket$ "realizing" $A$. This term usually is much shorter than the proof it came from, because in the process all subproofs of formulas without computational content can be ignored. The extracted term has a type $\tau(A)$, which depends on the logical shape of the proven formula $A$ only.

An important aspect of this "internal" term extraction (compared with say the extraction of OCaml programs in Coq (Letouzey, 2003)) is that one stays within the language of the logical theory, and hence - for a particular proof $M$ - can prove within the system that the extracted term indeed realizes the formula $A$ (the "Soundness Theorem").

Of course, there is a good reason to extract programs rather than terms: running programs is much faster than evaluating (closed) terms. However, the point just made is a strong argument for term extraction, particularly in safety critical applications. Moreover, as should become clear from what is done in the present paper, with some care one may well design proofs (and the underlying data types) in such a way that the extracted terms are short and easy to read and evaluate. One can then go on and (automatically) translate these terms into code of a functional programming language, for faster evaluation.

1.1. Data types, typed $\lambda$-terms. When formalizing mathematical arguments with a planned extraction of realizing terms in mind, it is clearly important to represent the underlying objects in an appropriate way.

In our subject of elementary constructive analysis it is tempting to start with groups, rings, fields etc. (as in (Geuvers et al., 2000; Cruz-Filipe, 2004)). However, it turned out that in such a general approach it is hard to control the computational content of the proofs, and hence its extracted terms. This does not mean that an abstract approach is impossible for our task, but for the moment we prefer the more "concrete" setup, with explicit constructions of the objects.

- Positive natural numbers are written in binary; we take them as generated from 1 by two successors $n \mapsto 2 n$ and $n \mapsto 2 n+1$. In the corresponding free algebra we have the constructors One, SZero and SOne.

- An integer is either a positive number, or zero, or a negative number. 
- A rational is a pair of an integer and a positive, written i\#n. Notice that equality of rationals is not the literal one, but given by the usual equivalence relation.

- A real is a pair of a Cauchy sequence of rationals and a modulus. We view the reals as a data type (i.e., no properties), with constructor RealConstr as M, whose components are written $\mathrm{x}$ seq and $\mathrm{x}$ mod. Within this data type we inductively define the predicate Real $\mathrm{x}$, meaning that $\mathrm{x}$ is a (proper) real.

- A continuous function is viewed as an element of a data type with constructor ContConstr, whose fields are written $f$ doml, $f$ domr (for the left and right end point of its domain), $f$ approx (for the approximating function), $f$ uMod (for the uniform Cauchy modulus) and $f$ uModCont (for the modulus of uniform continuity). Within this data type we have an inductively defined predicate Cont $f$, meaning that $f$ is a (proper) continuous function.

From this material we can now build typed $\lambda$ terms, as usual. They are terms in the sense of Gödel's $T$ (1958), that is, contain (structural) recursion operators for every data type (i.e., free algebra), with arbitrary value types. These terms are the basis of our logical (better: arithmetical) system, which contains an induction scheme (w.r.t. arbitrary formulas) for every data type.

1.2. Heyting arithmetic $H A^{\omega}$ in finite types. For our present purpose it suffices to only allow decidable atomic formulas. So the atomic formulas are atom $\left(r^{\text {boole }}\right)$, indicating that the argument is true. Here $r$ is a term of type boole, for instance of the form $=(s, t)$ with $=$ a constant of type nat $\Rightarrow$ nat $\Rightarrow$ boole. Notice that there is no need for (logical) falsity $\perp$, since we can take the atomic formula $F:=$ atom(ff) - called arithmetical falsity - built from the boolean constant ff instead. The formulas of $\mathrm{HA}^{\omega}$ are built from atomic ones by the connectives $\rightarrow, \forall, \wedge$ and $\exists$. We define negation $\neg A$ by $A \rightarrow F$.

We use Gentzen's natural deduction calculus for logical derivations consisting of the well-known rules $\rightarrow^{+}, \rightarrow^{-}, \forall^{+}$and $\forall^{-}$. It will be convenient to write derivations as terms, where the derived formula is viewed as the type of the term. This representation is known under the name Curry-Howard correspondence.

We give an inductive definition of derivation terms in table 1 , where for clarity we have written the corresponding derivations to the left. For the universal quantifier $\forall$ there is an introduction rule $\forall^{+} x$ and an elimination rule $\forall^{-}$, whose right premise is the term $r$ to be substituted. The rule $\forall^{+} x$ is subject to the following (Eigen-) variable condition: The derivation term $M$ of the premise $A$ should not contain any open assumption with $x$ as a free variable.

The logical axioms are the truth axiom $\mathrm{Ax}_{\mathbb{t}}$ : atom(tt), the introduction and elimination axioms $\exists^{+}$and $\exists^{-}$for existence and $\wedge^{+}, \wedge^{-}$for conjunction:

$$
\begin{aligned}
& \exists^{+}: \forall_{x}\left(A \rightarrow \exists_{x} A\right), \\
& \exists^{-}: \exists_{x} A \rightarrow \forall_{x}(A \rightarrow B) \rightarrow B \quad(x \notin \mathrm{FV}(B)), \\
& \wedge^{+}: A \rightarrow B \rightarrow A \wedge B,
\end{aligned}
$$




\begin{tabular}{|c|c|}
\hline derivation & term \\
\hline$u: A$ & $u^{A}$ \\
\hline $\begin{array}{l}{[u: A]} \\
\quad \mid M \\
\frac{B}{A \rightarrow B} \rightarrow^{+} u\end{array}$ & $\left(\lambda u^{A} M^{B}\right)^{A \rightarrow B}$ \\
\hline $\begin{array}{cl}\mid M & \mid N \\
A \rightarrow B & A\end{array} \rightarrow^{-}$ & $\left(M^{A \rightarrow B} N^{A}\right)^{B}$ \\
\hline $\begin{array}{l}\mid M \\
\frac{A}{\forall_{x} A} \forall^{+} x \quad \text { (with var.cond.) }\end{array}$ & $\left(\lambda x M^{A}\right)^{\forall_{x} A}$ (with var.cond.) \\
\hline $\begin{array}{c}\mid M \\
\frac{\forall_{x} A(x) \quad r}{A(r)} \forall^{-}\end{array}$ & $\left(M^{\forall_{x} A(x)} r\right)^{A(r)}$ \\
\hline
\end{tabular}

TABle 1. Derivation terms for $\rightarrow$ and $\forall$

$$
\wedge^{-}: A \wedge B \rightarrow(A \rightarrow B \rightarrow C) \rightarrow C,
$$

and the induction axioms, say for the booleans, the unary natural numbers, the positive natural numbers written in binary, and pairs:

$$
\begin{aligned}
& \operatorname{Ind}_{p, A}: A(\mathrm{tt}) \rightarrow A(\mathrm{ff}) \rightarrow \forall_{p^{\text {boole }}} A(p), \\
& \operatorname{Ind}_{n, A}: A(0) \rightarrow \forall_{n}(A(n) \rightarrow A(\mathrm{~S} n)) \rightarrow \forall_{n^{\text {nat }}} A(n), \\
& \operatorname{Ind}_{b, A}: A(1) \rightarrow \forall_{b}\left(A(b) \rightarrow A\left(\mathrm{~S}_{0} b\right)\right) \rightarrow \forall_{b}\left(A(b) \rightarrow A\left(\mathrm{~S}_{1} b\right)\right) \rightarrow \forall_{b^{\text {bin }}} A(b), \\
& \operatorname{Ind}_{x^{\rho \times \sigma}, A}: \forall_{y^{\rho}, z^{\sigma}} A(\langle y, z\rangle) \rightarrow \forall_{x^{\rho \times \sigma}} A(x) .
\end{aligned}
$$

The final axiom expresses that every object of a pair type is in fact a pair; it is sometimes called pair elimination axiom.

Using boolean induction $\operatorname{Ind}_{p, A}$ we can derive the arithmetical form of ex-falso-quodlibet, that is, $F \rightarrow$ atom $(p)$ (recall $F:=$ atom(ff)), and then $F \rightarrow A$ for arbitrary formulas $A$.

Let Heyting arithmetic $\mathrm{HA}^{\omega}$ in finite types be the theory based on the axioms above including the induction axioms, and $\mathrm{ML}^{\omega}$ be the (many-sorted) minimal logic, where the induction axioms are left out. 
1.3. The type of a realizer. We assign to every formula $A$ an object $\tau(A)$ (a type or the "nulltype" symbol $\varepsilon$ ). $\tau(A)$ is intended to be the type of the program to be extracted from a proof of $A$. In case $\tau(A)=\varepsilon$ proofs of $A$ have no computational content; such formulas $A$ are called Harrop formulas. The definition can be conveniently written if we extend the use of $\rho \Rightarrow \sigma$ and $\rho \times \sigma$ to the nulltype symbol $\varepsilon$ :

$$
\begin{array}{rlrl}
(\rho \Rightarrow \varepsilon) & :=\varepsilon, & & (\rho \times \varepsilon):=\rho, \\
(\varepsilon \Rightarrow \sigma):=\sigma, & (\varepsilon \times \sigma):=\sigma, \\
(\varepsilon \Rightarrow \varepsilon):=\varepsilon, & (\varepsilon \times \varepsilon):=\varepsilon .
\end{array}
$$

With this understanding of $\rho \Rightarrow \sigma$ and $\rho \times \sigma$ we can simply write

$$
\begin{array}{rlrl}
\tau(P(\vec{s})) & :=\varepsilon, & & \\
\tau\left(\exists_{x^{\rho}} A\right) & :=(\rho \times \tau(A)), & \tau(A \wedge B) & :=(\tau(A) \times \tau(B)), \\
\tau\left(\forall_{x^{\rho}} A\right) & :=(\rho \Rightarrow \tau(A)), & \tau(A \rightarrow B):=(\tau(A) \Rightarrow \tau(B)) .
\end{array}
$$

1.4. Extracted terms. We define the extracted term $\llbracket M \rrbracket$, for a derivation $M$ using the axioms $\exists^{ \pm}, \wedge^{ \pm}$and induction axioms.

Assume first that $M$ derives a formula $A$ with $\tau(A) \neq \varepsilon$. Then its extracted term $\llbracket M \rrbracket$ of type $\tau(A)$ is

$$
\begin{aligned}
\llbracket u^{A} \rrbracket & :=x_{u}^{\tau(A)} \quad\left(x_{u}^{\tau(A)} \text { uniquely associated with } u^{A}\right), \\
\llbracket \lambda u^{A} M \rrbracket & := \begin{cases}\llbracket M \rrbracket & \text { if } \tau(A)=\varepsilon \\
\lambda x_{u}^{\tau(A)} \llbracket M \rrbracket & \text { otherwise, }\end{cases} \\
\llbracket M^{A \rightarrow B} N \rrbracket & := \begin{cases}\llbracket M \rrbracket & \text { if } \tau(A)=\varepsilon \\
\llbracket M \rrbracket \llbracket N \rrbracket & \text { otherwise, }\end{cases} \\
\llbracket\left(\lambda x^{\rho} M\right)^{\forall}{ }^{\forall} \rrbracket & :=\lambda x^{\rho} \llbracket M \rrbracket, \\
\llbracket M^{\forall_{x} A} t \rrbracket & :=\llbracket M \rrbracket t .
\end{aligned}
$$

We also need extracted terms for the axioms mentioned above; these will be defined below. For derivations $M^{A}$ where $\tau(A)=\varepsilon$ (i.e., $A$ is a Harrop formula) we define $\llbracket M \rrbracket:=\varepsilon$ ( $\varepsilon$ some new "nullterm" symbol).

For the axioms $\exists^{ \pm}, \wedge^{ \pm}$the extracted terms are easy to write down. The extracted term of an induction axiom is defined to be the corresponding recursion operator. For example, for the induction scheme

$$
\operatorname{Ind}_{n, A}: A(0) \rightarrow \forall_{n}(A(n) \rightarrow A(n+1)) \rightarrow \forall_{n} A(n)
$$

we have

$$
\llbracket \operatorname{Ind}_{n, A} \rrbracket:=\mathcal{R}_{\text {nat }}^{\tau}: \tau \Rightarrow(\text { nat } \Rightarrow \tau \Rightarrow \tau) \Rightarrow \text { nat } \Rightarrow \tau,
$$

where $\tau:=\tau(A) \neq \varepsilon$.

1.5. Realizability. We define the notion of (modified) realizability. The term "modified" is used for historical reasons, to distinguish this form of realizability from the (earlier) Kleene-style realizability. More precisely, we define formulas $r \mathrm{mr} A$, where $A$ is a formula and $r$ is either a term of type 
$\tau(A)$ if the latter is a type, or the symbol $\varepsilon$ if $\tau(A)=\varepsilon$.

$$
\begin{aligned}
& r \operatorname{mr} P(\vec{s}):=P(\vec{s}), \\
& r \operatorname{mr}\left(\exists_{x} A(x)\right):=\left\{\begin{array}{ll}
\varepsilon \mathrm{mr} A(r) & \text { if } \tau(A)=\varepsilon \\
r 1 \mathrm{mr} A(r 0) & \text { otherwise, }
\end{array} \quad(r 0, r 1 \text { components of } r)\right. \\
& r \operatorname{mr}\left(\forall_{x} A\right):= \begin{cases}\forall_{x} \varepsilon \mathrm{mr} A & \text { if } \tau(A)=\varepsilon \\
\forall_{x} r x \mathrm{mr} A & \text { otherwise, }\end{cases} \\
& r \operatorname{mr}(A \wedge B):= \begin{cases}(\varepsilon \mathrm{mr} A) \wedge(r \mathrm{mr} B) & \text { if } \tau(A)=\varepsilon \\
(r \mathrm{mr} A) \wedge(\varepsilon \mathrm{mr} B) & \text { if } \tau(B)=\varepsilon \\
(r 0 \mathrm{mr} A) \wedge(r 1 \mathrm{mr} B) & \text { otherwise, }\end{cases} \\
& r \operatorname{mr}(A \rightarrow B):= \begin{cases}\varepsilon \mathrm{mr} A \rightarrow r \mathrm{mr} B & \text { if } \tau(A)=\varepsilon \\
\forall_{x}(x \mathrm{mr} A \rightarrow \varepsilon \mathrm{mr} B) & \text { if } \tau(A) \neq \varepsilon=\tau(B) \\
\forall_{x}(x \mathrm{mr} A \rightarrow r x \mathrm{mr} B) & \text { otherwise. }\end{cases}
\end{aligned}
$$

The $\exists$-free formulas play a special role in this context. Their crucial property is that for an $\exists$-free formula $A$ we have $(\varepsilon \operatorname{mr} A)=A$. Notice also that every formula $r \operatorname{mr} A$ is $\exists$-free.

The natural question under what conditions a formula $A$ and its modified realizability interpretation $\exists_{x} x \mathrm{mr} A$ are equivalent has been answered by Troelstra. For the formulation we need the axioms of choice and independence of premise. The axiom of choice (AC) is the scheme

$$
\forall_{x^{\rho}} \exists_{y^{\sigma}} A(x, y) \rightarrow \exists_{f^{\rho} \Rightarrow \sigma} \forall_{x^{\rho}} A(x, f(x)) .
$$

The independence of premise scheme $\left(\mathrm{IP}_{\mathcal{C}}\right)$, relative to a set $\mathcal{C}$ of formulas, is

$$
\left(A \rightarrow \exists_{x^{\rho}} B\right) \rightarrow \exists_{x^{\rho}}(A \rightarrow B) \text { with } A \in \mathcal{C} \text { and } x \notin \mathrm{FV}(A) .
$$

We will need ( $\operatorname{IP}_{\exists \text {-free}}$ ) for the set of $\exists$-free formulas, and $\left(\operatorname{IP}_{\varepsilon}\right)$ for the set of Harrop formulas.

Theorem (Characterization; cf. (Troelstra, 1973, 3.4.8)).

$$
\mathrm{HA}^{\omega}+\mathrm{AC}+\mathrm{IP}_{\exists-\text { free }} \vdash A \leftrightarrow \exists_{x} x \mathrm{mr} A .
$$

The direction " $\leftarrow$ " can be proved in $\mathrm{ML}^{\omega}$ alone, provided $A$ has only $\exists$-free premises.

Proof. Induction on $A$; we only treat the case $A \rightarrow B$ with $\tau(A) \neq \varepsilon$ and $\tau(B) \neq \varepsilon$.

$$
\begin{aligned}
(A \rightarrow B) & \leftrightarrow\left(\exists_{x} x \mathrm{mr} A \rightarrow \exists_{y} y \mathrm{mr} B\right) & & \text { by } \mathrm{IH} \\
& \leftrightarrow \forall_{x}\left(x \mathrm{mr} A \rightarrow \exists_{y} y \mathrm{mr} B\right) & & \text { by } \mathrm{ML}^{\omega} \\
& \leftrightarrow \forall_{x} \exists_{y}(x \mathrm{mr} A \rightarrow y \mathrm{mr} B) & & \text { by }\left(\mathrm{IP}_{\exists-\text { free }}\right) \\
& \leftrightarrow \exists_{f} \forall_{x}(x \mathrm{mr} A \rightarrow f(x) \mathrm{mr} B) & & \text { by }(\mathrm{AC}) \\
& \leftrightarrow \exists_{f} f \mathrm{mr}(A \rightarrow B) . & &
\end{aligned}
$$


Now assume that we only have $\exists$-free premises.

$$
\begin{aligned}
\exists_{y} y \operatorname{mr}(A \rightarrow B) & \rightarrow \exists_{y}(\varepsilon \mathrm{mr} A \rightarrow y \mathrm{mr} B) \\
& \rightarrow \exists_{y}(A \rightarrow y \mathrm{mr} B) \\
& \rightarrow\left(A \rightarrow \exists_{y} y \mathrm{mr} B\right) \\
& \rightarrow(A \rightarrow B),
\end{aligned}
$$

by IH.

By induction on derivations one can prove (cf. (Troelstra, 1973, 3.4.5))

Theorem (Soundness). Let $M$ be a derivation

$$
\mathrm{HA}^{\omega}+\mathrm{AC}+\mathrm{IP}_{\varepsilon} \vdash A
$$

from assumptions $u_{i}: C_{i}(i=1, \ldots, n)$. Then we can find a derivation $\mu(M)$

$$
\mathrm{HA}^{\omega} \vdash \llbracket M \rrbracket \mathrm{mr} A
$$

from assumptions $\bar{u}_{i}: x_{u_{i}} \operatorname{mr} C_{i}$.

Recall that $(\varepsilon \operatorname{mr} A)=A$ for every $\exists$-free formula $A$. So for a formula $\forall_{x^{\rho}} \exists_{y^{\sigma}} A(x, y)$ with $\exists$-free $A(x, y)$ we have $\tau\left(\forall_{x} \exists_{y} A(x, y)\right)=(\rho \Rightarrow \sigma)$ and

$$
t \text { mr } \forall_{x} \exists_{y} A(x, y)=\forall_{x} A(x, t x) .
$$

Hence as a corollary to the Soundness Theorem we immediately obtain the following. Let $M$ be a closed derivation $\mathrm{HA}^{\omega}+\mathrm{AC}+\mathrm{IP}_{\varepsilon} \vdash \forall_{x} \exists_{y} A(x, y)$, where $A(x, y)$ is $\exists$-free. Then $\mathrm{HA}^{\omega} \vdash \forall_{x} A(x, \llbracket M \rrbracket(x))$. Using the Characterization Theorem, we can extend this observation to arbitrary formulas $\forall_{x} \exists_{y} A(x, y)$. Clearly we can also add arbitrary Harrop formulas as axioms.

Theorem (Extraction). Assume

$$
\mathrm{HA}^{\omega}+\mathrm{AC}+\mathrm{IP}_{\varepsilon}+\mathrm{Ax}_{\varepsilon} \vdash \forall_{x} \exists_{y} A(x, y)
$$

with $A(x, y)$ an arbitrary formula containing at most the displayed variables free. Then we can find a closed $\mathrm{HA}^{\omega}$-term $t$ such that

$$
\mathrm{HA}^{\omega}+\mathrm{AC}+\mathrm{IP}_{\exists \text {-free }}+\mathrm{Ax}_{\varepsilon} \vdash \forall_{x} A(x, t x) .
$$

In fact,

$$
t= \begin{cases}\lambda x \cdot \llbracket M \rrbracket x 0 & \text { if } \tau(A(x, y)) \neq \varepsilon \\ \llbracket M \rrbracket & \text { otherwise. }\end{cases}
$$

Proof. We assume $\tau(A(x, y)) \neq \varepsilon$; otherwise the proof is even easier. $\mathrm{HA}^{\omega}+$ $\mathrm{Ax}_{\varepsilon}$ proves

$$
\begin{aligned}
& \llbracket M \rrbracket \operatorname{mr} \forall_{x} \exists_{y} A(x, y) \quad \text { by the Soundness Theorem } \\
& \forall_{x}\left(\llbracket M \rrbracket x \operatorname{mr} \exists_{y} A(x, y)\right) \\
& \forall_{x}(\llbracket M \rrbracket x 1 \mathrm{mr} A(x, \llbracket M \rrbracket x 0)) \\
& \forall_{x} \exists_{y} y \mathrm{mr} A(x, \llbracket M \rrbracket x 0) .
\end{aligned}
$$

Hence $\mathrm{HA}^{\omega}+\mathrm{AC}+\mathrm{IP}_{\exists-\text { free }}+\mathrm{Ax}_{\varepsilon} \vdash \forall_{x} A(x, \llbracket M \rrbracket x 0)$ by the Characterization Theorem. 


\section{INVERSE FunCTIONS}

We prove that every continuous function with a uniform modulus of increase has a continuous inverse. The proof below is based on a particular concept of a continuous function, as a type-1 object (using separability of the real numbers).

The setup of constructive analysis is essentially the one of Bishop (1967), and is only sketched here. More detailed elaborations can be found in (Andersson, 2001; Schwichtenberg, 2006).

We view a real $x$ as a Cauchy sequence $\left(a_{n}\right)_{n}$ of rationals with a separately given modulus $M$.

Definition. A real number $x$ is a pair $\left(\left(a_{n}\right)_{n \in \mathbb{N}}, M\right)$ with $a_{n} \in \mathbb{Q}$ and $M: \mathbb{N} \rightarrow \mathbb{N}$ such that $\left(a_{n}\right)_{n}$ is a Cauchy sequence with modulus $M$, that is

$$
\left|a_{n}-a_{m}\right| \leq 2^{-k} \text { for } n, m \geq M(k) .
$$

and $M$ is weakly increasing. $M$ is called Cauchy modulus of $x$.

We shall loosely speak of a real $\left(a_{n}\right)_{n}$ if the Cauchy modulus $M$ is clear from the context or inessential. Every rational $a$ is tacitly understood as the real represented by the constant sequence $a_{n}=a$ with the constant modulus $M(k)=0$.

When comparing two reals, $x<y$ needs a witness, but $x \leq y$ doesn't; in fact, we can prove $x \nless y \leftrightarrow y \leq x$. For reals $x=\left(\left(a_{n}\right)_{n}, M\right)$ and $y:=\left(\left(b_{n}\right)_{n}, N\right)$, define $x<_{k} y$ to mean $1 / 2^{k} \leq b_{p}-a_{p}$, for $p:=\max (M(k+$ 2), $N(k+2))$.

Constructively we cannot compare two reals, but we can compare a real with a proper interval:

Lemma (ApproxSplit). Let $x, y, z$ be given and assume $x<y$. Then either $z \leq y$ or $x \leq z$.

Proof. Let $x:=\left(\left(a_{n}\right)_{n}, M\right), y:=\left(\left(b_{n}\right)_{n}, N\right), z:=\left(\left(c_{n}\right)_{n}, L\right)$. Assume $x<_{k} y$, and let $q:=\max (p, L(k+2))$ and $d:=\left(b_{p}-a_{p}\right) / 4$.

Case $c_{q} \leq \frac{a_{p}+b_{p}}{2}$. We show $z \leq y$. It suffices to prove $c_{n} \leq b_{n}$ for $n \geq q$. To see this, observe

$$
c_{n} \leq c_{q}+\frac{1}{2^{k+2}} \leq \frac{a_{p}+b_{p}}{2}+\frac{b_{p}-a_{p}}{4}=b_{p}-\frac{b_{p}-a_{p}}{4} \leq b_{p}-\frac{1}{2^{k+2}} \leq b_{n}
$$

Case $c_{q} \leq \frac{a_{p}+b_{p}}{2}$. We show $x \leq z$, via $a_{n} \leq c_{n}$ for $n \geq q$.

$$
a_{n} \leq a_{p}+\frac{1}{2^{k+2}} \leq a_{p}+\frac{b_{p}-a_{p}}{4} \leq \frac{a_{p}+b_{p}}{2}-\frac{b_{p}-a_{p}}{4} \leq c_{q}-\frac{1}{2^{k+2}} \leq c_{n} .
$$

A continuous function $f: I \rightarrow \mathbb{R}$ on a compact interval $I$ with rational end points is given by

(a) an approximating map $h_{f}:(I \cap \mathbb{Q}) \times \mathbb{N} \rightarrow \mathbb{Q}$ and a map $\alpha_{f}: \mathbb{N} \rightarrow \mathbb{N}$ such that $\left(h_{f}(a, n)\right)_{n}$ is a Cauchy sequence with (uniform) modulus $\alpha_{f}$;

(b) a modulus $\omega_{f}: \mathbb{N} \rightarrow \mathbb{N}$ of (uniform) continuity, which satisfies

$$
|a-b| \leq 2^{-\omega_{f}(k)+1} \rightarrow\left|h_{f}(a, n)-h_{f}(b, n)\right| \leq 2^{-k} \quad \text { for } n \geq \alpha_{f}(k) ;
$$


$\alpha_{f}$ and $\omega_{f}$ are required to be weakly increasing. One may also add a lower bound $N_{f}$ and an upper bound $M_{f}$ for all $h_{f}(a, n)$.

Notice that a continuous function is given by objects of type level $\leq 1$ only. This is due to the fact that it suffices to define its values on rational numbers.

To prove the Intermediate Value Theorem, we begin with an auxiliary lemma, which from a "correct" interval $c<d$ (that is, $f(c) \leq 0 \leq f(d)$ and $\left.2^{-n} \leq d-c\right)$ constructs a new one $c_{1}<d_{1}$ with $d_{1}-c_{1}=\frac{2}{3}(d-c)$.

We say that $l \in \mathbb{N}$ is a uniform modulus of increase for $f:[a, b] \rightarrow \mathbb{R}$ if for all $c, d \in[a, b]$ and all $m \in \mathbb{N}$

$$
2^{-m} \leq d-c \rightarrow f(c)<_{m+l} f(d) .
$$

Lemma (IVTAux). Let $f:[a, b] \rightarrow \mathbb{R}$ be continuous, and with a uniform modulus $l$ of increase. Assume $a \leq c<d \leq b$, say $2^{-n}<d-c$, and $f(c) \leq 0 \leq f(d)$. Then we can construct $c_{1}, d_{1}$ with $d_{1}-c_{1}=\frac{2}{3}(d-c)$, such that again $a \leq c \leq c_{1}<d_{1} \leq d \leq b$ and $f\left(c_{1}\right) \leq 0 \leq f\left(d_{1}\right)$.

Proof. Let $c_{0}=c+\frac{d-c}{3}=\frac{2 c+d}{3}$ and $d_{0}=c+\frac{2(d-c)}{3}=\frac{c+2 d}{3}$. From $2^{-n}<d-c$ we obtain $2^{-n-2} \leq d_{0}-c_{0}$, so $f\left(c_{0}\right)<_{n+2+l} f\left(d_{0}\right)$. Now compare 0 with this proper interval, using ApproxSplit. In the first case we have $0 \leq f\left(d_{0}\right)$; then let $c_{1}=c$ and $d_{1}=d_{0}$. In the second case we have $f\left(c_{0}\right) \leq 0$; then let $c_{1}=c_{0}$ and $d_{1}=d$.

Theorem (IVT). If $f:[a, b] \rightarrow \mathbb{R}$ is continuous with $f(a) \leq 0 \leq f(b)$, and with a uniform modulus of increase, then we can find $x \in[a, b]$ such that $f(x)=0$.

Proof. Iterating the construction in the auxiliary lemma IVTAux above, we construct two sequences $\left(c_{n}\right)_{n}$ and $\left(d_{n}\right)_{n}$ of rationals such that for all $n$

$$
\begin{aligned}
& a=c_{0} \leq c_{1} \leq \cdots \leq c_{n}<d_{n} \leq \cdots \leq d_{1} \leq d_{0}=b, \\
& f\left(c_{n}\right) \leq 0 \leq f\left(d_{n}\right), \\
& d_{n}-c_{n}=(2 / 3)^{n}(b-a) .
\end{aligned}
$$

Let $x, y$ be given by the Cauchy sequences $\left(c_{n}\right)_{n}$ and $\left(d_{n}\right)_{n}$ with the obvious modulus. As $f$ is continuous, $f(x)=0=f(y)$ for the real number $x=y$.

From the Intermediate Value Theorem we obtain

Theorem (Inv). Let $f:[a, b] \rightarrow \mathbb{R}$ be continuous with a uniform modulus of increase, and assume $f(a) \leq a^{\prime}<b^{\prime} \leq f(b)$. We can find a continuous $g:\left[a^{\prime}, b^{\prime}\right] \rightarrow \mathbb{R}$ such that $f(g(y))=y$ for every $y \in\left[a^{\prime}, b^{\prime}\right]$ and $g(f(x))=x$ for every $x \in[a, b]$ such that $a^{\prime} \leq f(x) \leq b^{\prime}$.

Proof. Let $f:[a, b] \rightarrow \mathbb{R}$ be continuous with a uniform modulus of increase, that is, some $l \in \mathbb{N}$ such that for all $c, d \in[a, b]$ and all $m \in \mathbb{N}$

$$
2^{-m} \leq d-c \rightarrow f(c)<_{m+l} f(d) .
$$

Let $f(a) \leq a^{\prime}<b^{\prime} \leq f(b)$. We construct a continuous $g:\left[a^{\prime}, b^{\prime}\right] \rightarrow \mathbb{R}$.

Let $u \in\left[a^{\prime}, b^{\prime}\right]$ be rational. Using $f(a)-u \leq a^{\prime}-u \leq 0$ and $0 \leq b^{\prime}-u \leq$ $f(b)-u$, the IVT gives us an $x$ such that $f(x)-u=0$, as a Cauchy sequence 
$\left(c_{n}\right)$. Let $h_{g}(u, n):=c_{n}$. We can easily define the modulus $\alpha_{g}$ such that for $n \geq \alpha_{g}(k),(2 / 3)^{n}(b-a) \leq 2^{-\omega_{f}(k+l+2)}$ : let

$$
\alpha_{g}(k):=2\left(k_{1}+\omega_{f}(k+l+2)\right) \text { with } k_{1} \text { such that } b-a \leq 2^{k_{1}} .
$$

For the uniform modulus $\omega_{g}$ of continuity assume $a^{\prime} \leq u<v \leq b^{\prime}$ and $k \in \mathbb{N}$. We claim that with $\omega_{g}(k):=k+l+2$ ( $l$ from the hypothesis on the slope) we can prove the required property

$$
|u-v| \leq 2^{-\omega_{g}(k)+1} \rightarrow\left|h_{g}(u, n)-h_{g}(v, n)\right| \leq 2^{-k} \quad\left(n \geq \alpha_{g}(k)\right) .
$$

Let $a^{\prime} \leq u<v \leq b^{\prime}$ and $n \geq \alpha_{g}(k)$. For $c_{n}^{(u)}:=h_{g}(u, n)$ and $c_{n}^{(v)}:=h_{g}(v, n)$ assume that $\left|c_{n}^{(u)}-c_{n}^{(v)}\right|>2^{-k}$; we must show $|u-v|>2^{-\omega_{g}(k)+1}$.

By the proof of the Intermediate Value Theorem we have

$$
d_{n}^{(u)}-c_{n}^{(u)} \leq(2 / 3)^{n}(b-a) \leq 2^{-\omega_{f}(k+l+2)} \text { for } n \geq \alpha_{g}(k) .
$$

Using $f\left(c_{n}^{(u)}\right)-u \leq 0 \leq f\left(d_{n}^{(u)}\right)-u$, the fact that a continuous function $f$ has $\omega_{f}$ as a modulus of uniform continuity gives us

$$
\left|f\left(c_{n}^{(u)}\right)-u\right|=u-f\left(c_{n}^{(u)}\right) \leq f\left(d_{n}^{(u)}\right)-f\left(c_{n}^{(u)}\right) \leq 2^{-k-l-2}
$$

and similarly $\left|f\left(c_{n}^{(v)}\right)-v\right| \leq 2^{-k-l-2}$. Hence, using $\left|f\left(c_{n}^{(u)}\right)-f\left(c_{n}^{(v)}\right)\right|>2^{-k-l}$ (which follows from $\left|c_{n}^{(u)}-c_{n}^{(v)}\right|>2^{-k}$ by the hypothesis on the slope),

$|u-v| \geq\left|f\left(c_{n}^{(u)}\right)-f\left(c_{n}^{(v)}\right)\right|-\left|f\left(c_{n}^{(u)}\right)-u\right|-\left|f\left(c_{n}^{(v)}\right)-v\right|>2^{-k-l-1}=2^{-\omega_{g}(k)+1}$.

So we have a continuous $g:\left[a^{\prime}, b^{\prime}\right] \rightarrow \mathbb{R}$.

The equation $f(g(u))=u$ now follows from $u=f(x)$ and the fact that $g(u)$ is represented by the sequence $h_{g}(u, n)=c_{n}$, and $x$ is given by $\left(c_{n}\right)$ as well. Since continuous functions are determined by their values on the rationals, we have $f(g(y))=y$ for $y \in\left[a^{\prime}, b^{\prime}\right]$.

For every $x \in[a, b]$ with $a^{\prime} \leq f(x) \leq b^{\prime}$, from $g(f(x))<x$ we obtain the contradiction $f(x)=f(g(f(x)))<f(x)$ by the hypothesis on the slope, and similarly for $>$. Using $u \nless v \leftrightarrow v \leq u$ we obtain $g(f(x))=x$.

As an example, consider the squaring function $f:[1,2] \rightarrow[1,4]$, given by the approximating map $h_{f}(a, n):=a^{2}$, constant Cauchy modulus $\alpha_{f}(k):=$ 1 , and modulus $\omega_{f}(k):=k+3$ of uniform continuity. The modulus of increase is $l:=0$, because for all $c, d \in[1,2]$

$$
2^{-m} \leq d-c \rightarrow c^{2}<_{m} d^{2} .
$$

Then $h_{g}(u, n):=c_{n}^{(u)}$, as constructed in the IVT for $x^{2}-u$, iterating IVTAux. The Cauchy modulus is $\alpha_{g}(k)=2(k+5)$, and the modulus of uniform continuity is $\omega_{g}(k):=k+2$.

\section{Formalization}

We now aim at formalizing the proof above, with the planned extraction of realizing terms in mind. The formalization itself is (tedious but) straightforward; proof scripts are available at www.minlog-system.de, in the directory examples/analysis. We do not give details here, but rather comment on some crucial issues that need to be addressed when one wants to machine extract realizers whose evaluation can be done efficiently. 
3.1. Computation rules. Computable functionals are defined by "computation rules" (Berger et al., 2003; Berger, 2005b); these rules are added to the standard conversion rules of typed $\lambda$-calculus. To simplify equational reasoning, terms with the same normal form are identified.

A system of computation rules for a defined constant $D$ consists of finitely many equations $D \vec{P}_{i}=Q_{i}(i=1, \ldots, n)$ with constructor patterns $\vec{P}_{i}$, such that $\vec{P}_{i}$ and $\vec{P}_{j}(i \neq j)$ are non-unifiable. Constructor patterns are lists of applicative terms with distinct variables, defined inductively as follows (we write $\vec{P}(\vec{x})$ to indicate all variables in $\vec{P}$; all expressions must be typecorrect):

- $x(x)$ is a constructor pattern.

- If $C$ is a constructor and $\vec{P}(\vec{x})$ a constructor pattern, then $(C \vec{P})(\vec{x})$ is a constructor pattern.

- If $\vec{P}(\vec{x})$ and $Q(\vec{y})$ are constructor patterns whose variables $\vec{x}$ and $\vec{y}$ are disjoint, then $(\vec{P}, Q)(\vec{x}, \vec{y})$ is a constructor pattern.

Important examples are the (Gödel) structural recursion operators. However, in practice one wants to define computable functionals by recursion equations, and if possible consider total functionals only. This can be achieved if the patterns on the lhs are "complete" (as for the structural recusion operators) and moreover the rules terminate (as for Gödel's T (1958)). Then every closed term of ground type reduces to a "numeral" (or a "canonical term"), that is, a term built from constructors only.

For example, addition for rational numbers is defined by the computation rule converting $(\mathrm{i} 1 \# \mathrm{k} 1)+(\mathrm{i} 2 \# \mathrm{k} 2)$ into $\mathrm{i} 1 * \mathrm{k} 2+\mathrm{i} 2 * \mathrm{k} 1 \# \mathrm{k} 1 * \mathrm{k} 2$.

3.2. External code as part of arithmetical constants. A problem when computing on rationals with the rule above is that the gcd is not cancelled out automatically. Therefore we add "external code" to the internal representation of the function. It works as follows: whenever addition for rationals is called with numerical arguments, these arguments are converted into Scheme rationals, then added with the rational addition function of Scheme, and the result is converted back into the internal representation (using the \#-constructor) of a rational.

3.3. Cleaning of reals. After some computations involving real numbers it is to be expected that the rational numbers occurring in the Cauchy sequences may become rather complex. Hence under computational aspects it is necessary to be able to clean up a real, as follows.

Lemma. For every real $x=\left(\left(a_{n}\right)_{n}, M\right)$ we can construct an equivalent real $y=\left(\left(b_{n}\right)_{n}, N\right)$ where the rationals $b_{n}$ are of the form $c_{n} / 2^{n}$ with integers $c_{n}$, and with modulus $N(k)=k+2$.

Proof. Let $c_{n}:=\left\lfloor a_{M(n)} \cdot 2^{n}\right\rfloor$ and $b_{n}:=c_{n} \cdot 2^{-n}$, hence

$$
\frac{c_{n}}{2^{n}} \leq a_{M(n)}<\frac{c_{n}}{2^{n}}+\frac{1}{2^{n}} \quad \text { with } c_{n} \in \mathbb{Z} .
$$


Then for $m \leq n$

$$
\begin{aligned}
\left|b_{m}-b_{n}\right| & =\left|c_{m} \cdot 2^{-m}-c_{n} \cdot 2^{-n}\right| \\
& \leq\left|c_{m} \cdot 2^{-m}-a_{M(m)}\right|+\left|a_{M(m)}-a_{M(n)}\right|+\left|a_{M(n)}-c_{n} \cdot 2^{-n}\right| \\
& \leq 2^{-m}+2^{-m}+2^{-n} \\
& <2^{-m+2},
\end{aligned}
$$

hence $\left|b_{m}-b_{n}\right| \leq 2^{-k}$ for $n \geq m \geq k+2=: N(k)$, so $\left(b_{n}\right)_{n}$ is a Cauchy sequence with modulus $N$.

To prove that $x$ is equivalent to $y:=\left(\left(b_{n}\right)_{n}, N\right)$, observe

$$
\begin{aligned}
\left|a_{n}-b_{n}\right| & \leq\left|a_{n}-a_{M(n)}\right|+\left|a_{M(n)}-c_{n} \cdot 2^{-n}\right| \\
& \leq 2^{-k-1}+2^{-n} \quad \text { for } n, M(n) \geq M(k+1) \\
& \leq 2^{-k} \quad \text { if in addition } n \geq k+1 .
\end{aligned}
$$

Hence $\left|a_{n}-b_{n}\right| \leq 2^{-k}$ for $n \geq \max (k+1, M(k+1))$, and therefore $x=y$.

3.4. Removal of duplicated parts in terms. In machine generated terms (e.g., those obtained by term extraction) it often happens that a subterm has many occurrences in a term, which leads to unwanted recomputations when evaluating it. A possible cure is to "optimize" the term after extraction, and replace for instance $M[x:=N]$ with many occurrences of $x$ in $M$ by $(\lambda x M) N$ (or a corresponding "let"-expression). However, this can already be done at the proof level: When an object (value of a variable or realizer of a premise) might be used more than once, make sure (if necessary by a cut) that the goal has the form $A \rightarrow B$ or $\forall_{x} A$. Now use the "identity lemma" Id $: \hat{P} \rightarrow \hat{P}$, whose predicate variable $\hat{P}$ is then instantiated with $A \rightarrow B$ or $\forall_{x} A$; its realizer has the form $\lambda f, x . f x$. However, if Id is not "animated" (see Section 4.1 below), the extracted term has the form $\operatorname{cId}(\lambda x M) N$, which is printed as [let $x \quad N M$ ].

3.5. Quantifiers without computational content. Besides the usual quantifiers, $\forall$ and $\exists$, Minlog has so-called non-computational quantifiers, $\forall^{\text {nc }}$ and $\exists^{\text {nc }}$, which allow for the extraction of simpler terms. The nc-quantifiers, which were first introduced by Berger (1993), can be viewed as a refinement of the Set/Prop distinction in constructive type systems like Coq or Agda. Intuitively, a proof of $\forall_{x}^{\text {nc }} A(x)(A(x)$ non-Harrop, i.e., with a strictly positive occurrence of an existential quantifier) represents a procedure that assigns to every $x$ a proof $M(x)$ of $A(x)$ where $M(x)$ does not make "computational use" of $x$, i.e., the extracted term $\llbracket M(x) \rrbracket$ does not depend on $x$. Dually, a proof of $\exists_{x}^{\text {nc }} A(x)$ is a proof of $M(x)$ for some $x$ where the witness $x$ is "hidden", that is, not available for computational use. Consequently, the types of extracted terms for nc-quantifiers are $\tau\left(\forall_{x^{\rho}}^{\mathrm{nc}} A\right)=\tau\left(\exists_{x^{\rho}}^{\mathrm{nc}} A\right)=\tau(A)$ as opposed to $\tau\left(\forall_{x^{\rho}} A\right)=(\rho \Rightarrow \tau(A))$ and $\tau\left(\exists_{x^{\rho}} A\right)=\rho \times \tau(A)$. The extraction rules are, for example in the case of $\forall^{\text {nc }}$-introduction and -elimination, $\llbracket\left(\lambda x \cdot M^{A(x)}\right)^{\forall x_{x}^{\text {nc }} A(x)} \rrbracket=\llbracket M \rrbracket$ and $\llbracket\left(M_{x}^{\forall_{x}^{\text {nc }} A(x)} r\right)^{A(r)} \rrbracket=\llbracket M \rrbracket$ as opposed to $\llbracket\left(\lambda x \cdot M^{A(x)}\right)^{\forall x A(x)} \rrbracket=\llbracket \lambda x M \rrbracket$ and $\llbracket\left(M^{\forall} A(x) r\right)^{A(r)} \rrbracket=\llbracket M r \rrbracket$. In order for the extracted terms to be correct the variable condition for $\forall^{\text {nc }}$-introduction needs to be strengthened by requiring in addition the abstracted variable $x$ not to occur in the extracted term $\llbracket M \rrbracket$. Note that for a Harrop formula 
$A$ the formulas $\forall_{x}^{\text {nc }} A$ and $\forall_{x} A$ are equivalent; similarly, $\exists_{x}^{\text {nc }} A$ and $\exists_{x} A$ are equivalent.

\section{Extracted terms}

For the formalization of and term extraction from a moderately complex proof (like the one of the Inversion Theorem above) it is indispensible to organize it into some lemmata, and then combine its extracted term from the ones for the lemmata. To make this work in practice, it is helpful to have a mechanism to "animate" or "deanimate" lemmata, or more precisely the constants that denote their computational content. We first describe this method, and then use it to build the extracted term of our theorem.

4.1. Animation. Suppose a proof of a theorem uses a lemma. Then the proof term contains just the name of the lemma, say L. In the term extracted from this proof we want to preserve the structure of the original proof as much as possible, and hence we use a new constant cL at those places where the computational content of the lemma is needed. When we want to execute the program, we have to replace the constant $\mathrm{CL}$ corresponding to a lemma $\mathrm{L}$ by the extracted program of its proof. This can be achieved by adding computation rules for CL and CGA. We can be rather flexible here and enable/block rewriting by using animate/deanimate as desired.

4.2. Extracted term of some auxiliary lemmata. The term extracted from the proof of ApproxSplit is

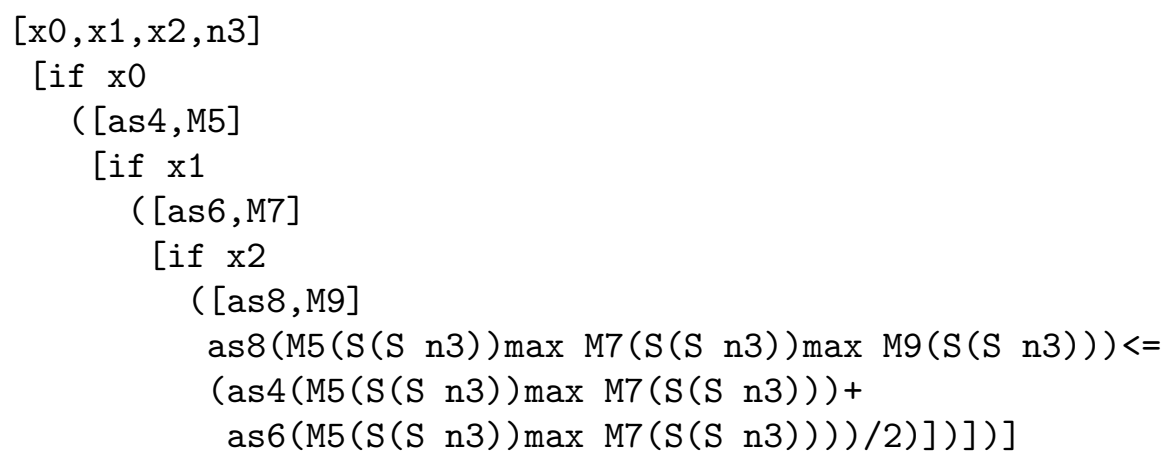

of type real=>real=>real=>pos=>boole. It takes three reals $x, y, z$ with moduli $M, N, K$ (here given by their Cauchy sequences as4, as6, as 8 and moduli M5, M7, M9) and a positive number $k$ (here $\mathrm{n} 3)$, and computes $p:=$ $\max (M(k+2), N(k+2))$ and $q:=\max (p, L(k+2))$. Then the choice whether to go right or left is by computing the boolean value $c_{q} \leq \frac{a_{p}+b_{p}}{2}$. Notice that the if-operator serves as a destructor here: it just takes the given real apart.

For the auxiliary lemma IVTAux we obtain the extracted term

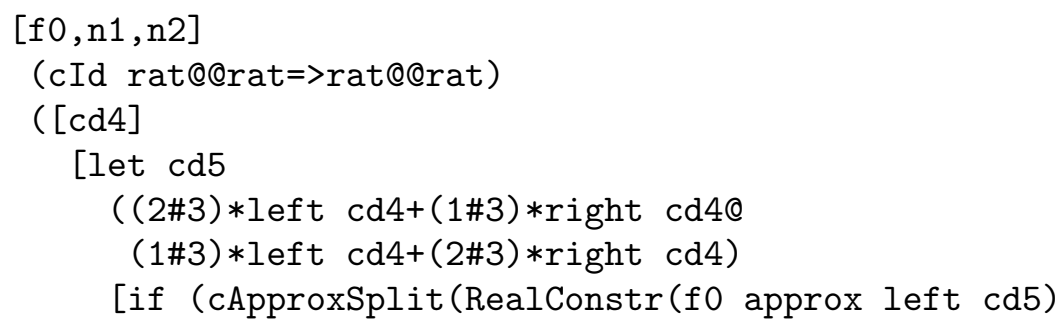


of type cont=>pos=>pos=>rat@@rat=>rat@@rat. As in the informal proof, it takes a continuous $f$ (here $\mathrm{f} 0$ ), a uniform modulus $l$ of increase (here $\mathrm{n} 1$ ), a positive number $n$ (here $\mathrm{n} 2$ ) and two rationals $c, d$ (here the pair cd4) such that $2^{-n}<d-c$. Let $c_{0}:=\frac{2 c+d}{3}$ and $d_{0}:=\frac{c+2 d}{3}$ (here the pair cd5, introduced via let because it is used four times). Then ApproxSplit is applied to $f\left(c_{0}\right), f\left(d_{0}\right), 0$ and the witness $n+2+l$ (here $\left.\mathrm{S}(\mathrm{S}(\mathrm{n} 2+\mathrm{n} 1))\right)$ for $f\left(c_{0}\right)<f\left(d_{0}\right)$. In the first case we go left, that is $c_{1}:=c$ and $d_{1}:=d_{0}$, and in the second case we go right, that is $c_{1}:=c_{0}$ and $d_{1}:=d$.

In the proof of the Intermediate Value Theorem, the construction step in IVTAux (from a pair $c, d$ to the "better" pair $c_{0}, d_{0}$ ) had to be iterated, to produce two sequences $\left(c_{n}\right)_{n}$ and $\left(d_{n}\right)_{n}$ of rationals. This is the content of a separate lemma IVTcds, whose extracted term is

$$
\begin{aligned}
{[\mathrm{f} 0, \mathrm{n} 1, \mathrm{n} 2] \text { (cDC rat@@rat) } } & \text { (f0 dom1@f0 domr) } \\
& \text { ([n4]cIVTAux f0 } \mathrm{n} 1(\mathrm{n} 2+\mathrm{n} 4))
\end{aligned}
$$

of type cont=>pos=>pos=>pos=>rat@@rat. It takes a continuous $f:[a, b] \rightarrow$ $\mathbb{R}$ (here $\mathrm{f} 0$ ), a uniform modulus $l$ of increase (here $\mathrm{n} 1$ ), and a positive number $k_{0}$ (here n2) such that $2^{-k_{0}}<b-a$. Then the axiom of dependent choice DC is used, to construct from an initial pair $\left(c_{0}, d_{0}\right)=(a, b)$ of rationals (here $f 0$ doml@fo domr) a sequence of pairs of rationals, by iterating the computational content cIVTAux of the lemma IVTAux.

4.3. Extracted term of the Inversion Theorem. The proof of the Inversion Theorem does not use the Intermediate Value theorem directly, but its essential ingredient IVTcds. Here is its extracted term, which constructs a continuous inverse:

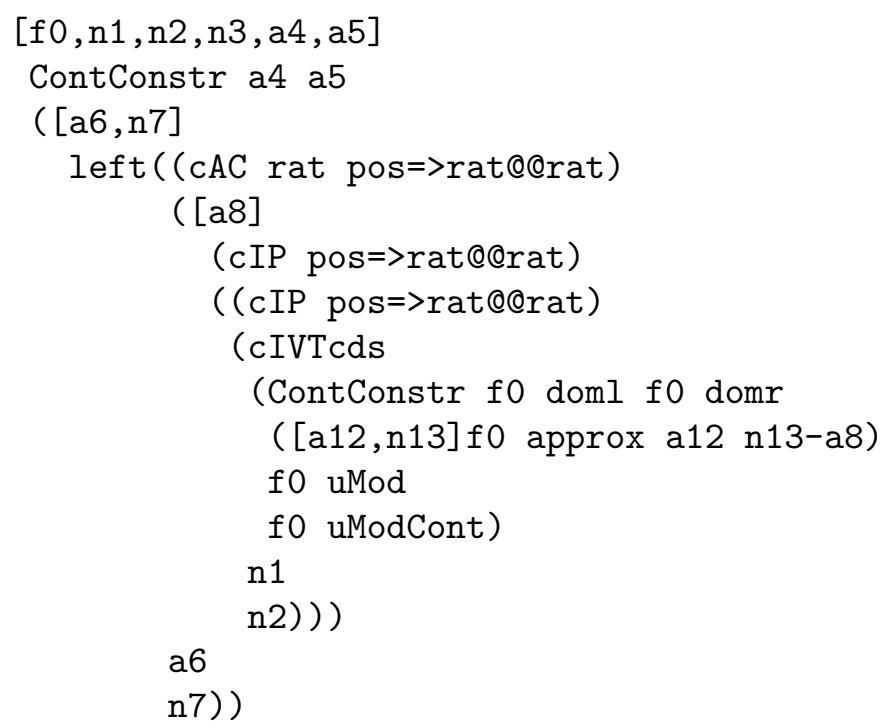


$([n 6]$ SZero $(f 0$ uModCont $(S(S(n 6+n 1))))+n 3+n 3)$

$([n 6] S(S(n 6+n 1)))$

It takes a continuous function $f$ (here $\mathrm{f} 0$ ), a uniform modulus $l$ of increase (here $\mathrm{n} 1$ ), positive numbers $k_{0}, k_{1}$ (here $\mathrm{n} 2, \mathrm{n} 3$ ) such that $2^{-k_{0}-1}<b-a<$ $2^{k_{1}}$ and two rationals $a_{1}<a_{2}$ (here a4, a5) in the range of $f$. Then the continuous inverse $g$ is constructed (via ContConstr) from

- an approximating map,

- a uniform Cauchy modulus (involving the modulus of uniform continuity coming with $f$ ), and

- an easy and explicit modulus of uniform continuity.

The approximating map takes $a, u$ (here $\mathrm{a} 6, \mathrm{n} 7$ ). Ignoring the computational content CAC, cIP of AC, IP (which are identities), it yields the left component (i.e., the Cauchy sequence) of the result of applying cIVTcds to a continuous function close to the original $f$.

4.4. Numerical approximations. To compute numerical approximations of values of an inverted function we need RealApprox, stating that every real can be approximated by a rational. Its extracted term is

$[\mathrm{x} 0, \mathrm{n} 1][$ if $\mathrm{x0}([\mathrm{as} 2, \mathrm{M} 3]$ as2 $(\mathrm{M} 3 \mathrm{n} 1))]$

of type real=>pos=>rat. It takes a real $x 0$ (here given by the Cauchy sequence as2 and modulus M3) and a positive number $k$ (here $\mathrm{n} 1$ ), and computes a rational $a$ such that $|x-a| \leq 2^{-k}$.

To compose Inv with RealApprox, we prove a proposition InvApprox stating that given an error bound, we can find a rational approximating the value of the inverted function $g$ up to this bound. Clearly we need to refer to this value and hence the inverted function $g$ in the statement of the theorem, but on the other hand we do not want to see a representation of $g$ in the extracted term, but only the construction of the rational approximation from the error bound. Therefore in the statement of InvApprox we use the non-computational quantifier $\exists^{\text {nc }}$ (see Section 3.5), for the inversion $g$ of the given continuous $f$. The extracted term of InvApprox then simply is

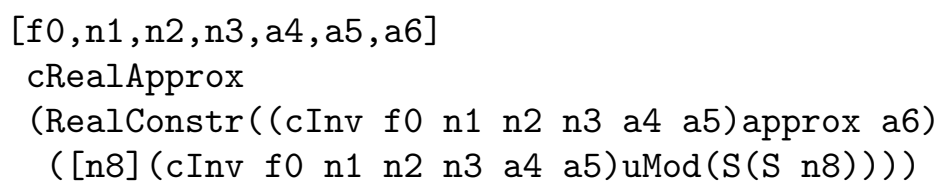

4.5. Animation of the auxiliary lemmas. Now we "animate" the auxiliary lemmas, that is, add computation rules for all constants with "c" in front of the name of the lemma. For InvApprox this gives






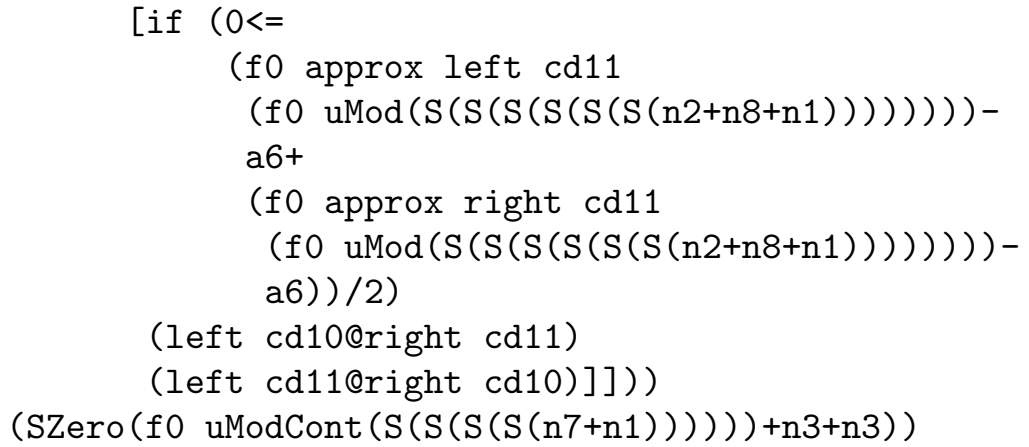

4.6. Numerical computations. We use this term to compute numerical approximations of values of an inverted function. First we construct the continuous function $x \mapsto x^{2}$ on [1,2], with its (trivial) uniform Cauchy modulus and modulus of uniform continuity, and give it the name sq:

(define sq (pt "ContConstr $12([\mathrm{a} 0, \mathrm{n} 1] \mathrm{a} 0 * \mathrm{a} 0)([\mathrm{n} 0] 1)([\mathrm{n}] \mathrm{n}+3) ")$ )

We now apply the extracted term of theorem InvApprox to

- the continuous sq to be inverted,

- a uniform modulus $l$ of increase,

- a positive number $k_{0}$ such that $2^{-k_{0}-1}<b-a$, and a positive number $k_{1}$ such that $b-a<2^{k_{1}}$ (which all happen to be 1 in this case),

- two rational bounds $a_{1}, b_{1}$ for an interval in the range,

and normalize the result:

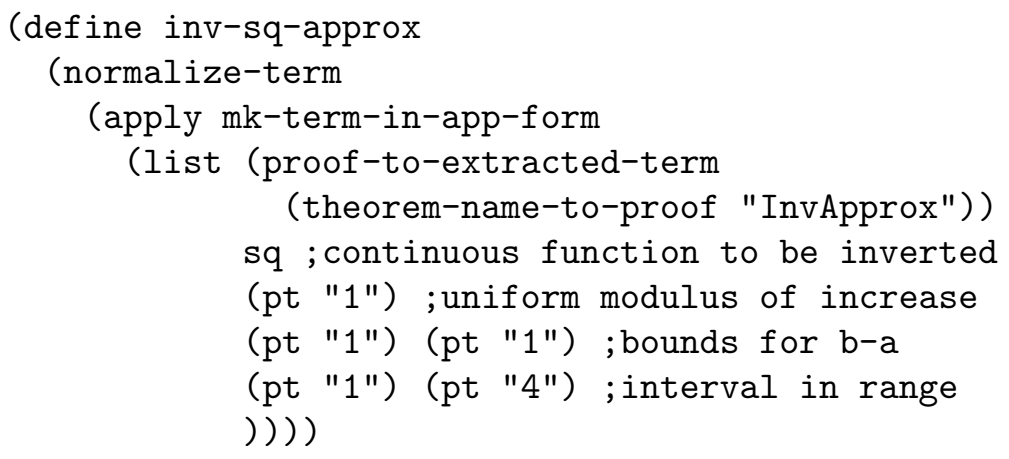

which prints as

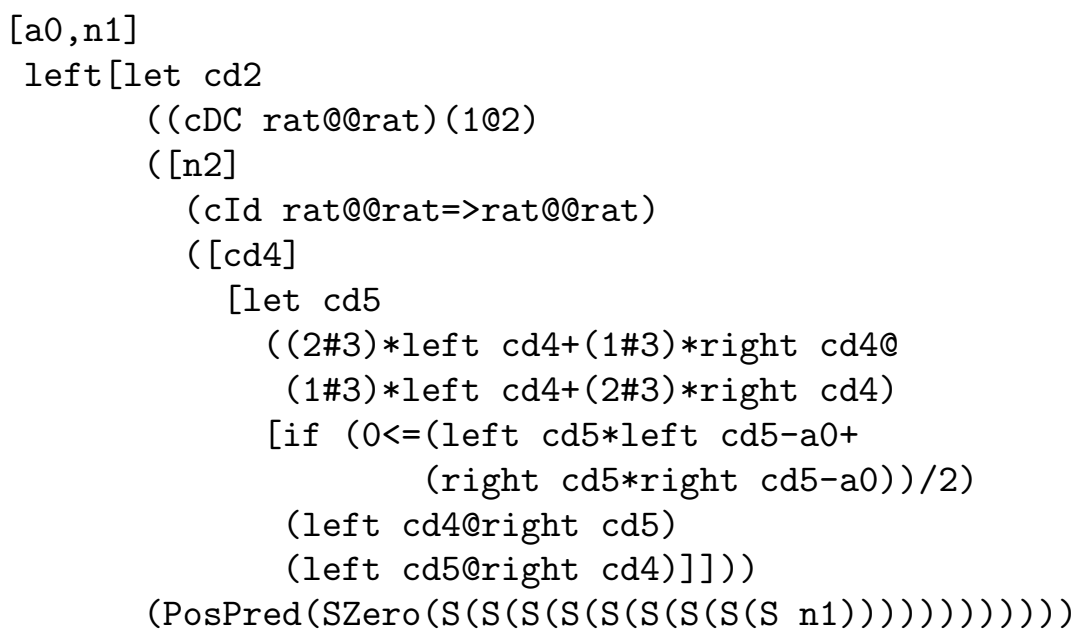




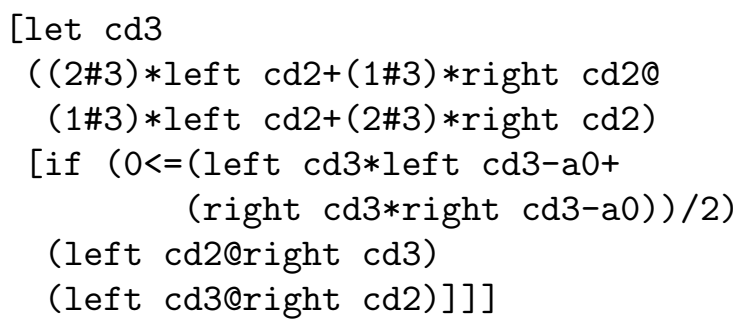

The term inv-sq-approx has type rat=>pos=>rat, where the first argument is for the rational to be inverted and the second argument $k$ is for the error bound $2^{-k}$. We can now directly (that is, without first translating into a programming language) use it to compute an approximation of say $\sqrt{3}$ to 20 binary digits. To do this, we need to "animate" Id and then normalize the result of applying inv-sq-approx to 3 and 20 (we use normalization by evaluation here, for efficiency reasons):

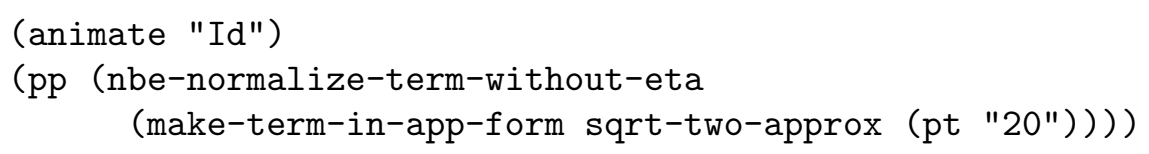

The result (returned in 2 seconds) is the rational

$2719394071183166419623547282 \# 1570042899082081611640534563$

or 1.7320508075117231 , which differs from $\sqrt{3}=1.7320508075688772$ at the 11-th (decimal) digit.

4.7. Translation into Scheme expressions. For a further speed-up (beyond the use of external code; cf. Section 3.2), we can also translate this internal term (where "internal" means "in our underlying logical language", hence usable in formal proofs) into an expression of a programming language (Scheme in our case), by evaluating (term-to-expr inv-sq-approx). This Scheme program is very close to the internal term displayed above; the internal constant CDC (computational content of the axiom of dependent choice) is replaced by the corresponding Scheme function (a curried form of iteration):

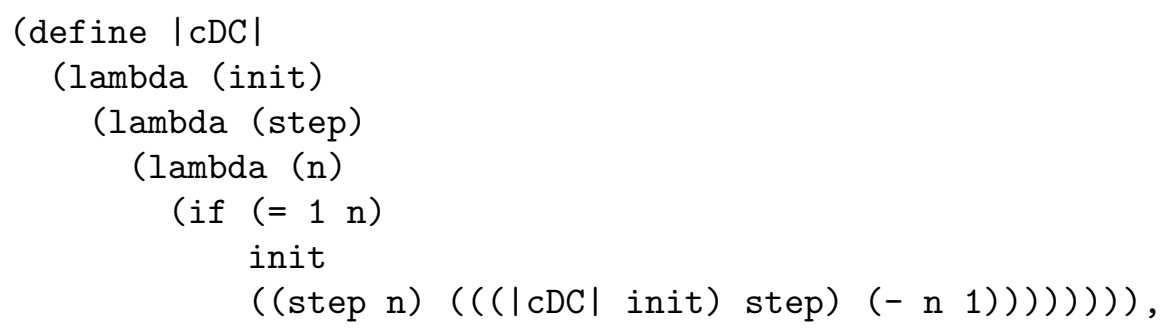

the internal arithmetical functions $+, *, /,<=$ by the ones from the programming language and the internal pairing and unpairing functions by cons, car and cdr. - It turns out that this code is reasonably fast: evaluating

(((ev (term-to-expr inv-sq-approx)) 3) 100)

gives the result in .5 seconds, with an accuracy of 100 binary digits. 


\section{Conclusion, Future WORK}

The present case study shows that it is possible - albeit after some formalization effort - to machine extract reasonable terms from proofs in constructive analysis, and that ordinary evaluation of these terms can be used to numerically compute approximations to say reals whose existence is claimed by the theorems, with a prescribed precision.

As for future work, an obvious canditate is to do the same for the CauchyEuler construction of approximate solutions to ordinary differential equations. A particularly promising candiate is the treatment of ordinary differential equations in Chapter 1 of Hurewicz's textbook (1958), which can easily be adapted to our constructive setting. It should also be possible to compare estimates for solutions of ordinary differential equations with the treatment of the same problem in the interval analysis setting of Moore (1966).

\section{REFERENCES}

P. Andersson. Exact real arithmetic with automatic error estimates in a computer algebra system. Master's thesis, Mathematics department, Uppsala University, 2001.

J. Berger. Exact calculation of inverse functions. Math. Log. Quart., 51(2): 201-205, 2005a.

U. Berger. Program extraction from normalization proofs. In M. Bezem and J. Groote, editors, Typed Lambda Calculi and Applications, volume 664 of LNCS, pages 91-106. Springer Verlag, Berlin, Heidelberg, New York, 1993.

U. Berger. Uniform Heyting Arithmetic. Annals Pure Applied Logic, 133: 125-148, 2005b.

U. Berger, M. Eberl, and H. Schwichtenberg. Term rewriting for normalization by evaluation. Information and Computation, 183:19-42, 2003.

E. Bishop. Foundations of Constructive Analysis. McGraw-Hill, New York, 1967.

L. Cruz-Filipe. Constructive Real Analysis: a Type-Theoretical Formalization and Applications. PhD thesis, Nijmegen University, 2004.

H. Geuvers, F. Wiedijk, and J. Zwanenburg. A constructive proof of the fundamental theorem of algebra without using the rationals. In P. Callaghan, Z. Luo, J. McKinna, and R. Pollack, editors, Proc. Types 2000, volume 2277 of LNCS, pages 96-111. Springer Verlag, Berlin, Heidelberg, New York, 2000.

K. Gödel. Über eine bisher noch nicht benützte Erweiterung des finiten Standpunkts. Dialectica, 12:280-287, 1958.

W. Hurewicz. Lectures on Ordinary Differential Equations. MIT Press, Cambridge, Mass., 1958.

U. Kohlenbach. Real growth in standard parts of analysis. Habilitationsschrift, Fachbereich Mathematik, Universität Frankfurt am Main, 1995.

G. Kreisel. Interpretation of analysis by means of constructive functionals of finite types. In A. Heyting, editor, Constructivity in Mathematics, pages 101-128. North-Holland, Amsterdam, 1959. 
P. Letouzey. A New Extraction for Coq. In H. Geuvers and F. Wiedijk, editors, Types for Proofs and Programs, Second International Workshop, TYPES 2002, volume 2646 of Lecture Notes in Computer Science. Springer-Verlag, 2003.

M. Mandelkern. Continuity of monotone functions. Pacific J. of Math., 99 (2):413-418, 1982.

R. E. Moore. Interval Analysis. Prentice-Hall, 1966.

H. Schwichtenberg. Constructive analysis with witnesses. In Schwichtenberg and Spies (2006), pages 323-353.

H. Schwichtenberg and K. Spies, editors. Proof Technology and Computation. Proc. NATO Advanced Study Institute, Marktoberdorf, 2003, volume 200 of Series III: Computer and Systems Sciences, 2006. IOS Press.

A. S. Troelstra, editor. Metamathematical Investigation of Intuitionistic Arithmetic and Analysis, volume 344 of Lecture Notes in Mathematics. Springer Verlag, Berlin, Heidelberg, New York, 1973. 